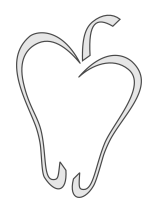

ISSN $1852-4249$

\title{
An efficient impurity-solver for the dynamical mean field theory algorithm
}

\author{
Y. Núñez Fernández, ${ }^{1 *}$ K. Hallberg ${ }^{1}$
}

\begin{abstract}
One of the most reliable and widely used methods to calculate electronic structure of strongly correlated models is the Dynamical Mean Field Theory (DMFT) developed over two decades ago. It is a non-perturbative algorithm which, in its simplest version, takes into account strong local interactions by mapping the original lattice model on to a single impurity model. This model has to be solved using some many-body technique. Several methods have been used, the most reliable and promising of which is the Density Matrix Renormalization technique. In this paper, we present an optimized implementation of this method based on using the star geometry and correction-vector algorithms to solve the related impurity Hamiltonian and obtain dynamical properties on the real frequency axis. We show results for the half-filled and doped one-band Hubbard models on a square lattice.
\end{abstract}

\section{Introduction}

Materials with strongly correlated electrons have attracted researchers in the last decades. The fact that most of them show interesting emergent phenomena like superconductivity, ferroelectricity, magnetism, metal-insulator transitions, among other properties, has triggered a great deal of research.

The presence of strongly interacting local orbitals that causes strong interactions among electrons makes these materials very difficult to treat theoretically. Very successful methods to calculate electronic structure of weakly correlated materials, such as the Density Functional Theory (DFT) [1], lead to wrong results when used in some of these systems. The DFT-based local density approxima-

*E-mail: yurielnf@gmail.com

1 Centro Atómico Bariloche and Instituto Balseiro, CNEA, CONICET, Avda. E. Bustillo 9500, 8400 San Carlos de Bariloche, Río Negro, Argentina tion (LDA) [2] and its generalizations are unable to describe accurately the strong electron correlations. Also, other analytical methods based on perturbations are no longer valid in this case so other methods had to be envisaged and developed.

More than two decades ago, the Dynamical Mean Field Theory (DMFT) was developed to study these materials. This method and its successive improvements $[3-8]$ have been successful in incorporating the electronic correlations and more reliable calculations were done. The combination of the DMFT with LDA allowed for band structure calculations of a large variety of correlated materials (for reviews, see Refs. $[9,10]$ ), where the DMFT accounts more reliably for the local correlations $[11,12]$.

The DMFT relies on the mapping of the correlated lattice onto an interacting impurity for which the fermionic environment has to be determined self-consistently until convergence of the local Green's function and the local self-energy is reached. This approach is exact for the infinitely 
Papers in Physics, vol. 9, ART. 090005 (2017) / Y. Núñez Fernández et al.

coordinated system (infinite dimensions), the noninteracting model and in the atomic limit. Therefore, the possibility to obtain reliable DMFT solutions of lattice Hamiltonians relies directly on the ability to solve (complex) quantum impurity models.

Since the development of the DMFT, several quantum impurity solvers were proposed and used successfully; among these, we can mention the iterated perturbation theory (IPT) $[13,14]$, exact diagonalization (ED) [15], the Hirsch-Fye quantum Monte Carlo (HFQMC) [16], the continuous time quantum Monte Carlo (CTQMC) [17-20], noncrossing approximations (NCA) [21], and the numerical renormalization group (NRG) [22,23]. All of these methods imply certain approximations. For a more detailed description, see [24].

Some years ago, we proposed the Density Matrix Renormalization Group (DMRG) as a reliable impurity-solver [25-27] which allows to surmount some of the problems existing in other solvers, giving, for example, the possibility of calculating dynamical properties directly on the real frequency axis. Other related methods followed, such as in $[28,29]$. This way, more accurate results can be obtained than, for example, using algorithms based on Monte Carlo techniques. The scope of this paper is to detail the implementation of this method and to show recent applications and potential uses.

\section{DMFT in the square lattice}

We will consider the Hubbard model on a square lattice:

$$
H=t \sum_{\langle i j\rangle \sigma} c_{i \sigma}^{\dagger} c_{j \sigma}+U \sum_{i} n_{i \uparrow} n_{i \downarrow}-\mu \sum_{i} n_{i}
$$

where $c_{i \sigma}\left(c_{i \sigma}^{\dagger}\right)$ annihilates (creates) an electron with spin $\sigma=\uparrow, \downarrow$ at site $i, n_{i \sigma}=c_{i \sigma}^{\dagger} c_{i \sigma}$ is the density operator, $n_{i}=n_{i \downarrow}+n_{i \uparrow}, U$ is the Coulomb repulsion, $\mu$ is the chemical potential, and $\langle i j\rangle$ represents nearest neighbor sites.

Changing to the Bloch basis $d_{\mathbf{k}}^{\dagger}$, the noninteracting part becomes:

$$
H^{0}=\sum_{k, \sigma} t(\mathbf{k}) d_{\mathbf{k} \sigma}^{\dagger} d_{\mathbf{k} \sigma}
$$

with $t(\mathbf{k})=2 t\left(\cos k_{x}+\cos k_{y}\right)-\mu$. The Green's function for (1) is hence given by:

$$
G(\mathbf{k}, \omega)=[\omega-t(\mathbf{k})-\Sigma(\mathbf{k}, \omega)]^{-1},
$$

where $\Sigma(\mathbf{k}, \omega)$ is the self-energy.

The DMFT makes a local approximation of $\Sigma(\mathbf{k}, \omega)$, that is, $\Sigma(\mathbf{k}, \omega) \approx \Sigma(\omega)$. This locality of the magnitudes allows us to map the lattice problem onto an auxiliar impurity problem that has the same local magnitudes $G(\omega)$ and $\Sigma(\omega)$. The impurity is coupled to a non-interacting bath, which should be determined iteratively. The Hamiltonian can be written:

$$
H_{i m p}=H_{l o c}+H_{b},
$$

where $H_{l o c}$ is the local part of (1)

$$
H_{l o c}=-\mu n_{0}+U n_{0 \uparrow} n_{0 \downarrow},
$$

and the non-interacting part $H_{b}$ representing the bath is:

$$
H_{b}=\sum_{i \sigma} \lambda_{i} b_{i \sigma}^{\dagger} b_{i \sigma}+\sum_{i \sigma} v_{i}\left[b_{i \sigma}^{\dagger} c_{0 \sigma}+H . c .\right]
$$

where $b_{i \sigma}^{\dagger}$ represents the creation operator for the bath-site $i$ and spin $\sigma$, label " 0 " corresponds to the interacting site.

The algorithm is summarized as:

(i) Start with $\Sigma(\omega)=0$.

(ii) Calculate the Green's function for the local interacting lattice site:

$$
\begin{aligned}
G(\omega) & =\frac{1}{N} \sum_{k} G(\mathbf{k}, \omega) \\
& =\frac{1}{N} \sum_{k}[\omega-t(\mathbf{k})-\Sigma(\omega)]^{-1} .
\end{aligned}
$$

(iii) Calculate the hybridization

$$
\Gamma(\omega)=\omega+\mu-\Sigma(\omega)-[G(\omega)]^{-1} .
$$

(iv) Find a Hamiltonian representation $H_{i m p}$ with hybridization $\Gamma_{d}(\omega)$ to approximate $\Gamma(\omega)$. The hybridization $\Gamma_{d}(z)$ is characterized by the parameters $v_{i}$ and $\lambda_{i}$ of $H_{i m p}$ through: 
Papers in Physics, vol. 9, ART. 090005 (2017) / Y. Núñez Fernández et al.

$$
\Gamma_{d}(\omega)=\sum_{i} \frac{v_{i}^{2}}{\omega-\lambda_{i}} .
$$

(v) Calculate the Green's function $G_{i m p}(\omega)$ at the impurity of the Hamiltonian $H_{i m p}$ using DMRG.

(vi) Obtain the self-energy

$$
\Sigma(\omega)=\omega+\mu-\left[G_{i m p}(\omega)\right]^{-1}-\Gamma_{d}(\omega) .
$$

Return to (ii) until convergence.

At step (iv) we should find the parameters $v_{i}$ and $\lambda_{i}$ by fitting the calculated hybridization $\Gamma(\omega)$ using expression (9). At half-filling, because of the electron-hole symmetry, we have $\Gamma(\omega)=\Gamma(-\omega)$ and hence $\lambda_{-i}=-\lambda_{i}$, and $v_{-i}=v_{i}$, where the bath index $i$ goes from $-p$ to $p$, and it does not include $i=0$ for an even number of bath sites $2 p$.

Almost all of the computational time is spent at step (v), where the dynamics of a single impurity Anderson model (SIAM) (see Fig. 1) is calculated. We use the correction-vector for DMRG following [30]. The one-dimensional representation of the problem (needed for a DMRG calculation) is as showed in Fig. 1, except that for the spin degree of freedom we duplicate the graph, generating two identical chains, one for each spin. Moreover, it should be noticed that this is not a local or shortrange 1D Hamiltonian (usually called chain geometry, where the DMRG is supposed to work very well). However, we refer to $[31,32]$ where strong evidence of better performance of the DMRG for this kind of geometry (star geometry) compared to chain geometry is presented.

The correction-vector for DMRG consists of targeting not only the ground state $\left|E_{0}\right\rangle$ of the system but also the correction-vector $\left|V_{i}\right\rangle$ associated to the frequency $\omega_{i}$ (and its neighborhood), that is:

$$
\left(\omega_{i}+i \eta-H_{i m p}-E_{0}\right)\left|V_{i}\right\rangle=c_{0}^{\dagger}\left|E_{0}\right\rangle,
$$

where a Lorentzian broadering $\eta$ is introduced to deal with the poles of a finite-length SIAM. For a better matching between the $\omega$ windows (with width approximately $\eta$ ), we target the correction vectors of the extremes of the window. Once the DMRG is converged, the Green's function is evaluated for a finer mesh (around 0.2 of the original

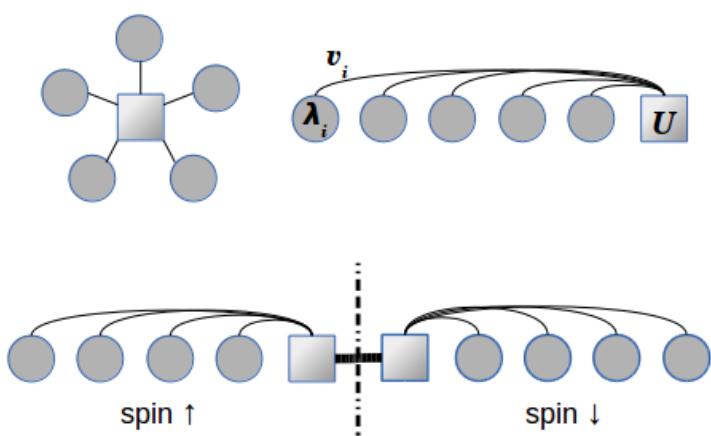

Figure 1: Schematic representation of the impurity problem for the DMFT. The circles (square) represent the non-interacting (interacting) sites, and the lines correspond to the hoppings. Top: star geometry drawn in two ways. Bottom: 1D representation as used for DMRG calculations.

window) [30]. In this way, a suitable renormalized representation of the operators is obtained to calculate the properties of the excitations around $\omega_{i}$, particularly the Green's function.

In what follows, we present results for a paradigmatic correlated model using the method described above.

\section{Results}

We have used this method to calculate the density of states (DOS) of the Hamiltonian (Eq. 1) on a square lattice with unit of energy $t=0.25$, for several dopings, given by the chemical potential. We consider a discarded weight of $10^{-11}$ in the DMRG procedure for which a maximum of around $m=128$ states were kept, even for the largest systems (50 sites). For these large systems, the ground state takes around 20 minutes to converge and each frequency window, between 5 and 20 minutes. This is an indication of the good efficiency of the method.

The metal-insulator Mott's transition at halffilling is showed in Fig. 2. The transition occurs between $U=3$ and $U=4$. In Fig. 3, we observe that the metallic character of the bands remains robust under doping for a given value of the interaction, showing a weight transfer between the bands due to the correlations. The metallic character is also seen in the variation of the filling with $\mu$. 
Papers in Physics, vol. 9, ART. 090005 (2017) / Y. Núñez Fernández et al.
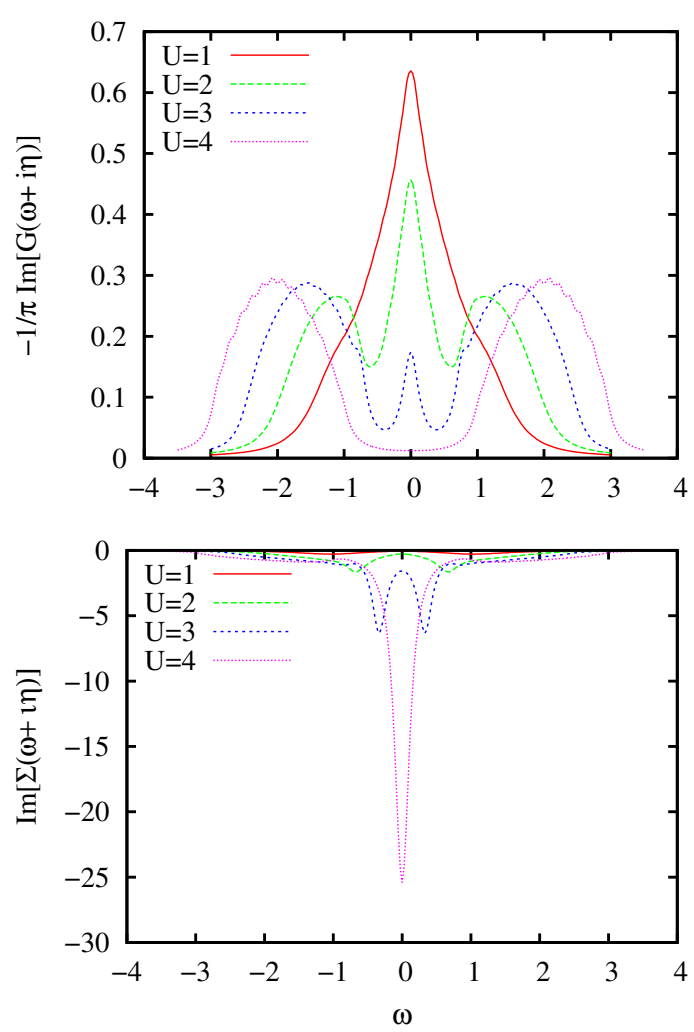

Figure 2: Top: Density of states for $U=1,2,3,4$ at half-filling. We use a bath with $30-50$ sites per spin and a Lorentzian broadening $\eta=0.12$. The Fermi energy is located at $\omega=0$. Bottom: Imaginary part of the self-energy.

Figure 4 shows our results for a larger value of the interaction $U$, for which we find a regime of doping having an insulating character. However, for a large enough doping (obtained for a large negative value of the chemical potential), the systems turn metallic and acquire a large density of states at the Fermi energy. While the system is insulating, changing the chemical potential only results in a rigid shift of the density of states. The small finite values of the DOS at the Fermi energy for the insulating cases are due to the Lorentzian broadening $\eta$, see Eq. (11).

\section{Conclusions}

We have presented here an efficient algorithm to calculate dynamical properties of correlated sys-
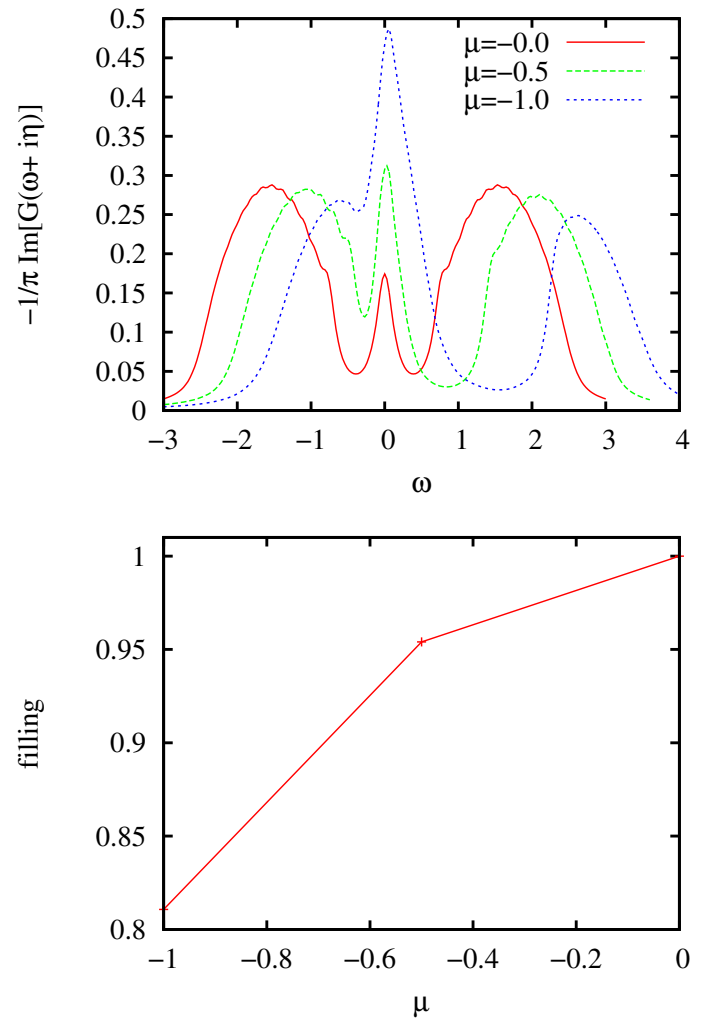

Figure 3: Top: Density of states for $U=3$, same parameters as in Fig. 2, and several chemical potentials ( $\mu=0$ corresponds to the half-filled case). Bottom: Filling vs chemical potential showing a metallic behavior.

tems such as the electronic structure for any doping. It is based on the Dynamical Mean Field theory method where we use the Density Matrix Renormalization Group (DMRG) as the impurity solver. By using the star geometry for the hybridization function (which reduces the entanglement enhancing the performance of the DMRG for larger bath sizes) together with the correction vector technique(which accurately calculates the dynamical response functions within the DMRG) we were able to obtain reliable real axis response functions, in particular, the density of states, for any doping, for the Hubbard model on a square lattice. This improvement will allow for the calculation of dynamical properties on the real energy axis for complex and more realistic correlated systems. 
Papers in Physics, vol. 9, ART. 090005 (2017) / Y. Núñez Fernández et al.
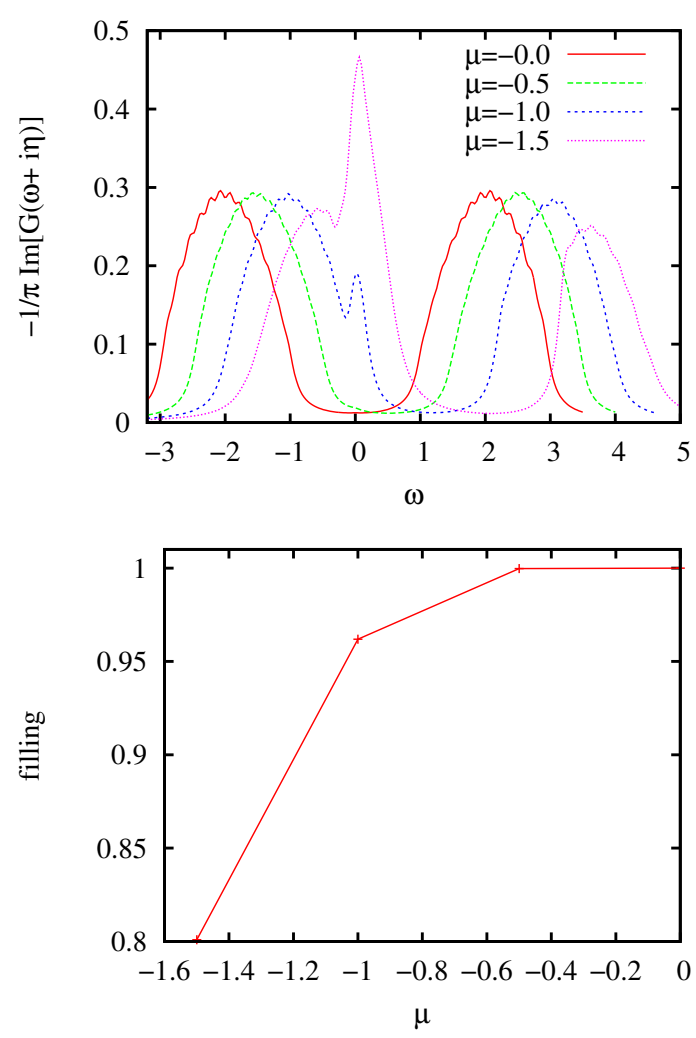

Figure 4: Top: Density of states for $U=4$, same parameters as in Fig. 2, and several chemical potentials ( $\mu=0$ corresponds to the half-filled case). Bottom: Filling vs chemical potential showing the transition from a metal to an insulator.

Acknowledgements - We thank Daniel García for useful discussions.

[1] P Hohenberg, W Kohn, Inhomogeneous electron gas, Phys. Rev. 136, B864 (1964).

[2] R O Jones, O Gunnarsson, The density functional formalism, its applications and prospects, Rev. Mod. Phys. 61, 689 (1989).

[3] G Kotliar, D Vollhardt, Strongly correlated materials: Insights from dynamical mean-field theory, Physics Today 57, 53 (2004).

[4] A Georges, G Kotliar, W Krauth, M J Rozenberg, Dynamical mean-field theory of strongly correlated fermion systems and the limit of in- finite dimensions, Rev. Mod. Phys. 68, 13 (1996).

[5] G Kotliar, S Y Savrasov, G Pálsson, G Biroli, Cellular dynamical mean field approach to strongly correlated systems, Phys. Rev. Lett. 87, 186401 (2001).

[6] T Maier, M Jarrell, T Pruschke, M H Hettler, Quantum cluster theories, Rev. Mod. Phys. 77, 1027 (2005).

[7] M H Hettler, A N Tahvildar-Zadeh, M Jarrell, T Pruschke, H R Krishnamurthy, Nonlocal dynamical correlations of strongly interacting electron systems, Phys. Rev. B 58, R7475 (1998).

[8] D Sénéchal, D Perez, M Pioro-Ladrière, Spectral weight of the Hubbard model through cluster perturbation theory, Phys. Rev. Lett. 84, 522 (2000).

[9] M Imada, T Miyake, Electronic structure calculation by first principles for strongly correlated electron systems, J. Phys. Soc. Jpn. 79, 112001 (2010).

[10] K Held, Electronic structure calculations using dynamical mean-field theory, Adv. in Phys. 56, 829 (2007).

[11] V I Anisimov, A I Poteryaev, M A Korotin, A O Anokhin, G Kotliar, First-principles calculations of the electronic structure and spectra of strongly correlated systems: dynamical mean-field theory, J. Phys. Condens. Mat. 9, 7359 (1997).

[12] A I Lichtenstein, M I Katsnelson, Ab initio calculations of quasiparticle band structure in correlated systems: $L D A++$ approach, Phys. Rev. B 57, 6884 (1998).

[13] A Georges, G Kotliar, Hubbard model in infinite dimensions, Phys. Rev. B 45, 6479 (1992).

[14] M J Rozenberg, G Kotliar, X Y Zhang, MottHubbard transition in infinite dimensions. II, Phys. Rev. B 49, 10181 (1994). 
Papers in Physics, vol. 9, ART. 090005 (2017) / Y. Núñez Fernández et al.

[15] M Caffarel, W Krauth, Exact diagonalization approach to correlated fermions in infinite dimensions: Mott transition and superconductivity, Phys. Rev. Lett. 72, 1545 (1994).

[16] J E Hirsch, R M Fye, Monte Carlo method for magnetic impurities in metals, Phys. Rev. Lett. 56, 2521 (1986).

[17] A N Rubtsov, V V Savkin, A I Lichtenstein, Continuous-time quantum Monte Carlo method for fermions, Phys. Rev. Lett. 72, 035122 (2005).

[18] P Werner, A Comanac, L de Medici, M Troyer, A J Millis, Continuous-Time Solver for Quantum Impurity Models, Phys. Rev. Lett. 97, 076405 (2006).

[19] H Park, K Haule, G Kotliar, Cluster Dynamical Mean Field Theory of the Mott Transition, Phys. Rev. Lett. 101, 186403 (2008).

[20] E Gull, A J Millis, A I Lichtenstein, A N Rubtsov, M Troyer, P Werner, Continuoustime Monte Carlo methods for quantum impurity models, Rev. Mod. Phys. 83, 349 (2011).

[21] T Pruschke, D L Cox, M Jarrell, Hubbard model at infinite dimensions: Thermodynamic and transport properties, Phys. Rev. Lett. 47, 3553 (1993).

[22] K G Wilson, The renormalization group: Critical phenomena and the Kondo problem, Rev. Mod. Phys. 47, 773 (1975).

[23] R Bulla, Zero temperature metal-insulator transition in the infinite-dimensional hubbard model, Phys. Rev. Lett. 83, 136 (1999);

R Bulla, A C Hewson, T Pruschke, Numerical renormalization group calculations for the self-energy of the impurity Anderson model, J. Phys. Condens. Mat. 10, 8365 (1998).

[24] K Hallberg, D J García, P Cornaglia, J Facio, Y Núñez-Fernández, State-of-the-art techniques for calculating spectral functions in models for correlated materials, EPL 112, 17001 (2015).
[25] D J García, K Hallberg, M J Rozenberg, Dynamical mean field theory with the density matrix renormalization group, Phys. Rev. Lett. 93, 246403 (2004).

[26] D J García, E Miranda, K Hallberg, M J Rozenberg, Mott transition in the Hubbard model away from particle-hole symmetry, Phys. Rev. B 75, 121102 (2007);

E Miranda, D J García, K Hallberg, M J Rozenberg, The metal-insulator transition in the paramagnetic Hubbard Model, Physica B: Cond. Mat. 403, 1465 (2008);

D J García, E Miranda, K Hallberg, M J Rozenberg, Metal-insulator transition in correlated systems: A new numerical approach, Physica B: Cond. Mat. 398, 407 (2007);

S Nishimoto, F Gebhard, E Jeckelmann, Dynamical density-matrix renormalization group for the Mott-Hubbard insulator in high dimensions, J. Phys. Condens. Mat. 16, 7063 (2004);

M Karski, C Raas, G Uhrig, Electron spectra close to a metal-to-insulator transition, Phys. Rev. B 72, 113110 (2005);

M Karski, C Raas, G Uhrig, Single-particle dynamics in the vicinity of the Mott-Hubbard metal-to-insulator transition, Phys. Rev. B 75, 075116 (2008);

C Raas, P Grete, G Uhrig, Emergent Collective Modes and Kinks in Electronic Dispersions, Phys. Rev. Lett. 102, 076406 (2009).

[27] Y Núñez Fernández, D García, K Hallberg, The two orbital Hubbard model in a square lattice: a DMFT + DMRG approach, J. Phys.: Conf. Ser. 568, 042009 (2014).

[28] M Ganahl et al, Efficient DMFT impurity solver using real-time dynamics with matrix product states, Phys. Rev. B 92, 155132 (2015).

[29] F Wolf, J Justiniano, I McCulloch, U Schollwöck, Spectral functions and time evolution from the Chebyshev recursion, Phys. Rev. B 91, 115144 (2015).

[30] T D Kühner, S R White, Dynamical correlation functions using the density matrix renormalization group, Phys. Rev. B 60, 335 (1999). 
PApers in Physics, vol. 9, ART. 090005 (2017) / Y. Núñez Fernández et al.

[31] A Holzner, A Weichselbaum, J von Delft, $M a-$ trix product state approach for a two-lead multilevel Anderson impurity model, Phys. Rev. B 81, 125126 (2010).
[32] F Alexander Wolf, I McCulloch, U Schollwöck, Solving nonequilibrium dynamical mean-field theory using matrix product states, Phys. Rev. B 90, 235131 (2014). 